\title{
Efficacy of Education, Continuous Monitoring and Nutritional Care on Quality of Life of Cirrhotic Patients and Reducing of Liver Cirrhosis Complications
}

\author{
Pezhman Alavinejad $^{1}$, Eskandar Hajiani ${ }^{1}$, Baharak Danyaee ${ }^{2}$, \\ Mehrnaz Morvaridi ${ }^{3}$ \\ ${ }^{1}$ Alimentary Tract Research Center, Ahvaz Jundishapur University of Medical Sciences, \\ Ahvaz, Iran \\ ${ }^{2}$ Internal Medicine Resident, Department of Internal Medicine, Ahvaz Jundishapur University of \\ Medical Sciences, Ahvaz, Iran \\ ${ }^{3}$ MSc Student of Nutrition Sciences, Alimentary Tract Research Center, Ahvaz Jundishapur \\ University of Medical Sciences, Ahvaz, Iran
}

Corresponding Author Pezhman Alavinejad

Mobile: 00989161115880

E mail: pezhmanalavinejad@g mail.com

Key words: education, liver cirrhosis, awareness, quality of life
Background and study aim: Liver cirrhosis is a complex chronic disease with a wide variety of morbidities and these complications directly affect the quality of life and result in significant morbidity and mortality. This study designed to determine the effects of an educational awareness program and continuous monitoring on quality of life (QOL) among cirrhotic patients and their complications.

Methods: Overall 18 cirrhotic patients included. An educational awareness program including a face-to-face training session, providing a guide booklet about caring of the liver cirrhosis, consulting with dietician and continuous monitoring of patients every other week was conducted and the patients followed for 6 months. The QOL, awareness score, complications of liver disease, severity of disease by using MELD score and laboratory parameters

\section{INTRODUCTION}

Liver cirrhosis by regeneration and fibrosis of the liver cells ultimately leads to portal hypertension and liver dysfunction [1]. This condition is a complex chronic disease with a wide variety of morbidities and estimated annual mortality rate about 5-10 cases per 100,000 people worldwide [2,3]. Nowadays, the most common causes of liver cirrhosis are alcohol-related liver disease (ARLD), non-alcoholic fatty liver disease (NAFLD) and viral hepatitis $[4,5]$.

Hepatic encephalopathy, ascites, gastrointestinal bleeding and portal were evaluated at baseline and at the end of intervention.

Results: At the end of the intervention, the prevalence of complications, including edema, ascites, hepatic encephalopathy, variceal bleeding and hospitalization rate were significantly decreased compare to baseline $(\mathrm{P}<0.05)$. The average scores of awareness and QOL improved significantly (52.78 \pm 20.36 to $88.89 \pm 10.49 ; \mathrm{P}=0.0001$ and $141.89 \pm 20.40$ vs. $182.72 \pm 10.27$; $\mathrm{P}=0.0001$ respectively). The laboratory parameters and the severity of the disease did not show any significant changes at the end of the study $(\mathrm{P}>0.05)$.

Conclusion: Using a simple educational program can significantly improve not only cirrhotics awareness and QOL but also reduce complications and duration of hospitalization which could be cost effective and worth to try among cirrhotics.

hypertension are the most common complications of liver cirrhosis [6]. These complications directly affect the quality of life and result in significant morbidity and mortality [7]. In addition, malnutrition is one of the known complications of the liver cirrhosis and has significant prognostic effect on irreversibility of this condition and its complications [8]. Liver cirrhosis management methods, including screening of hepatocellular carcinoma (HCC) and bleeding from gastrointestinal varices often focus on risk reduction. These strategies mostly target clinical factors and do not consider other patient related factors such as 
health-related quality of life (HRQOL). Measuring HRQOL can affect the disease management and is recognized as an important factor in chronic diseases such as liver cirrhosis [5].

Moreover, management of patients depends on their proper understanding of their condition and active participation in their care and decision making [9]. However, at the moment, patients' understanding and awareness about this condition is low and the reasons of this unawareness include: limited time for consultation in the clinic and the variable quality of educational resources such as websites and information leaflets [10,11]. The inherent complexity of liver cirrhosis and its complications may also limit access to information and understanding of the disease in some patients. However, there is little information and evidence about the patient's awareness about liver cirrhosis or the effectiveness of a routine educational intervention [13].

So it seems necessary to consider the importance of nutrition, counseling and awareness of patients about their disease, and its possible role for improving their quality of life. Moreover, its possible role in reducing the complications of liver cirrhosis should be emphasized. The current studies were limited to a specific range of cirrhotic patients with the same etiology as well as a certain stage of the disease according to the Child score, therefore, we conducted this study among patients who suffering liver cirrhosis with various causes and at various stages based on the MELD Score, to evaluate efficacy of close monitoring, education and nutritional support on the quality of life and their laboratory profile.

\section{METHODS}

This interventional cross sectional pilot study conducted on patients with liver cirrhosis who referred to the hepatology outpatient clinic of Ahvaz Imam Hospital in 2017. After being approved by the Ethics Committee of Ahvaz Jundishapur University of Medical Sciences (IRAJUMS. REC.1396.811), all over-18-year-old patients suffering from non-alcoholic liver cirrhosis were invited to participate without exclusion based on various stages of the Child Score. Then, 25 patients were invited to enter the study and informed consent was obtained from all of the participants. Exclusion criteria included: simultaneous medical conditions and synchronous diseases such as uncontrolled cardiopulmonary or renal disease, presence of any kind of malignancy such as hepatocellular carcinoma, pregnancy, and nonreferring or failure to follow after 6 months of intervention. This interventional study was performed on 25 cirrhotic patients, of which 7 were excluded from the study because of various reasons (death of the patient and lack of followup and or timely referral), and the final analysis were performed on 18 subjects. The study protocol conforms to the ethical guidelines of the 1975 Declaration of Helsinki as reflected in a priori approval by the institution's human research committee.

Before the intervention, the demographic information of each patient (including age, sex, education, marriage, etc.) and his or her clinical profile (including family history of liver disease, duration of illness, cause of illness plus results of laboratory parameters, complications of disease, quality of life, and knowledge of patients about their condition) were evaluated and recorded in each patient's special file.

The educational and awareness program delivered to all of the participants which include one-hour face-to-face awareness training session in a safe and relaxed environment. Educational content included some information about liver function, cirrhosis development with the progression of inflammation, risk factors, disease complications and management strategies. In addition, the patients were provided a booklet containing information about liver cirrhosis. Training about cirrhosis of the liver and its complications, diet, observing nutritional guidelines for reducing complications of the illness and hygiene was given to all of them. In this stage, a diet was also given to patients. In addition, they were contacted every two weeks and their questions and concerns about the disease were answered.

\section{Patient evaluation}

In all of the patients, the level of knowledge, quality of life, severity of illness, laboratory parameters (Na, K, BUN, Cr, CBC, ALT, AST, ALK P, INR, TSH, BIL) were evaluated at the beginning of the study and six months after the intervention (end of study). Body mass index (BMI) determined as $\mathrm{Kg} / \mathrm{m}^{2}$. The frequency of hospitalization and complications such as incidence of gastrointestinal bleeding, hepatic encephalopathy, severity of ascites and edema, and other complications such as electrolyte and 
laboratory abnormalities over a 6 months period were investigated, and they were compared with their same results during 6 months before of the study period.

Quality of life and disease severity assessment Severity of the liver cirrhosis was determined based on the Model for End-Stage Liver Disease (MELD) scale. The chronic liver disease questionnaire (CLDQ) was used to assess the quality of life of the patients [14]. This questionnaire has been widely used as a healthrelated quality of life assessment (HRQOL) tool and its validity has been confirmed in chronic liver disease with different etiologies and severity and in different languages [14-16]. The questionnaire consists of 29 questions in 6 sections: 8 questions about emotional functioning, 3 questions about abdominal symptoms, 3 questions about activity, 5 questions about systemic symptoms, 5 questions about fatigue, and 5 questions about anxiety. The questions are measured on a 7-point Likert-scale: Always 1; Very often 2; Mostly 3; Sometimes 4; Rarely 5 ; hardly ever 6; Never 7 scores. The final score of QOL obtained by calculating the total score of each individual. In general, each individual's score range varies between 29 and 203, and higher scores represent better QOL [14]. The validity of this questionnaire has been confirmed in previous studies in Iran [15] and in this study, the reliability of the quality of life questionnaire was assessed by calculating Cronbach's alpha (0.84).

\section{Awareness level}

A researcher-made questionnaire was used to measure patient's awareness about liver cirrhosis, consisting of 8 two-choice questions (yes 1; no $0)$. The awareness level of each person was obtained by calculating the total score of each individual. This level varies from 0 to 8 for them, and a higher score indicates more awareness among patients. Content validity of the questionnaire was evaluated by supervising of two well experienced hepatologists. Reliability of the questionnaire was calculated as 0.86 by using Cronbach's alpha.

\section{Statistical analysis}

SPSS version 22 was used for statistical analysis. Data were analyzed by descriptive statistics including mean, standard deviation, frequency and percentage. The normality of data was assessed by Kolmogorov-Smirnov test and homogeneity of variances by Leven test. Moreover, Wilcoxon singled-rank test was used to compare the mean of variables before and after intervention and chi-square test was used to compare qualitative variables. The significance level in the tests was considered to be 0.05 .

\section{RESULTS}

Average age of the participants was $47.00 \pm$ 17.42 y (18 to 83 years), which included 4 women and $14 \mathrm{men}$. The average duration of the disease from first diagnosis to participation in study was $2.52 \pm 2.32$ months ( 1 month to 9 years). The baseline characteristics of the patients are presented in Table (1).

After the completion of the study, the average time of participants hospitalization significantly decreased (Before the study $1.28 \pm 1.22$ days $(0$ to 4$)$ and at the end of the study $0.33 \pm 0.59(0-2)$ days, $\mathrm{P}=0.001$ ). Before study, $55.6 \%$ of patients had edema while after 6 months of close monitoring, this symptom decreased to $16.7 \%$. In addition, at the end of the study, the prevalence and incidence of bleeding from gastrointestinal varices and hepatic encephalopathy were also decreased. Hypothermia and serum potassium disturbance were not observed in any of the patients at the beginning and at the end of the intervention. Comparison of the liver cirrhosis complications before and after intervention is presented in Table (2).

As presented in Table 3, the laboratory values at the end of the study did not show any significant changes $(P>0.05)$. The severity of liver cirrhosis based on MELD score did not change significantly at the end of the study ( $\mathrm{P}>0.05)$ (Table 4).

As presented in Table 5, the level of patient's awareness before and after the intervention was $52.78 \pm 20.36$ and $88.89 \pm 10.40$, respectively (average $36.11 \%$ improve, $\mathrm{P}<0.0001$ ). The quality of life of the patients before and after the intervention was $69.9 \% \pm 10.05$ and $90.01 \%$, respectively ( $20.11 \%$ improvement). The dimensions of QOL including emotion, systemic and abdominal symptoms, activity, fatigue, and anxiety significantly improved at the end of the study $(\mathrm{P}<0.0001$, Fig. $1)$. 
Table (1) : Basic characteristics of the participants (BMI: Body Mass Index)

\begin{tabular}{|l|c|}
\hline \multicolumn{1}{|c|}{ Characteristics } & Frequency (percent) \\
\hline Sex & $4(22.2 \%)$ \\
Female & $14(77.8 \%)$ \\
Male & $3(16.7 \%)$ \\
\hline Marital status & $15(83.3 \%)$ \\
Single & \\
Married & \\
\hline Education & $2(11.1 \%)$ \\
Illiterate & $9(50.0 \%)$ \\
Sub diploma & $4(22.2 \%)$ \\
Diploma & $3(16.7 \%)$ \\
Academic education & $68.09 \pm 16.04(45-110)$ \\
\hline Weight (kg) & $170.00 \pm 10.49(152-187)$ \\
Height (cm) & $24.06 \pm 5.07(16.46-35.51)$ \\
BMI (Kg / m2) & $2(11.1 \%)$ \\
History of alcohol consumption & $6(33.3 \%)$ \\
\hline Smoking history & $3(16.7 \%)$ \\
\hline Family history of liver disease & $9(50 \%)$ \\
\hline Hospitalization history (yes) & \\
\hline Background of the underlying disease & $3(16.7 \%)$ \\
Diabetes Melitus & $2(11.1 \%)$ \\
Chronic kidney disease & $3(16.7 \%)$ \\
Others & \\
\hline Cause of disease & $8(44.4 \%)$ \\
Hepatitis C & $3(16.7)$ \\
Autoimmune hepatitis & $2(11.1)$ \\
Hepatitis B & $1(5.6)$ \\
Fatty Liver & $4(22.2)$ \\
Unknown & \\
\hline
\end{tabular}

Table (2) : Comparison of complications before and after intervention

\begin{tabular}{|l|c|c|c|}
\hline \multicolumn{1}{|c|}{ Complication of liver cirrhosis } & Before intervention & After intervention & P-value \\
\hline Ascites & $10(55.6 \%)$ & $2(11.1 \%)$ & 0.005 \\
Yes & $8(44.4 \%)$ & $16(88.9 \%)$ & \\
No & $8(44.4 \%)$ & $15(83.3 \%)$ & 0.002 \\
\hline Edema & $7(38.9 \%)$ & $3(16.7 \%)$ & 0 \\
No & $3(16.7 \%)$ & 0 & \\
Intensity of edema (+1) & $2(11.1 \%)$ & $18(100 \%)$ & 0.157 \\
Intensity of edema (+2) & $16(88.9 \%)$ & & \\
\hline Bleeding from gastrointestinal & & 0 & 0.157 \\
varices & $2(11.1 \%)$ & $18(100 \%)$ & \\
Yes & $16(88.9 \%)$ & & \\
No & & & \\
\hline Hepatic encephalopathy & & & \\
Yes & & & \\
No & & & \\
\end{tabular}

Alavinejad et al., Afro-Egypt J Infect Endem Dis 2019; 9(2): 167-175

https://aeji.journals.ekb.eg/

http://mis.zu.edu.eg/ajied/home.aspx 
Table (3) : Laboratory values before and at the end of the study

\begin{tabular}{|l|c|c|c|}
\hline \multicolumn{1}{|c|}{ Variable } & Before intervention & After the intervention & *P-value \\
\hline $\mathrm{BUN}(\mathrm{mg} / \mathrm{dL})$ & $16.12 \pm 10.5$ & $17.48 \pm 8.11$ & 0.397 \\
\hline $\mathrm{Cr}(\mathrm{mg} / \mathrm{dl})$ & $0.93 \pm 0.37$ & $1.09 \pm 0.36$ & 0.164 \\
\hline $\mathrm{Na}(\mathrm{mEq} / \mathrm{L})$ & $138.71 \pm 2.8$ & $139.58 \pm 2.71$ & 0.246 \\
\hline $\mathrm{K}(\mathrm{mEg} / \mathrm{L})$ & $4.02 \pm 0.38$ & $4.11 \pm 0.35$ & 0.135 \\
\hline $\mathrm{Hb}(\mathrm{g} / \mathrm{dL})$ & $11.73 \pm 2.36$ & $11.47 \pm 1.41$ & 0.34 \\
\hline $\mathrm{Ca}(\mathrm{mg} / \mathrm{dL})$ & $8.84 \pm 0.86$ & $9.08 \pm 0.24$ & 0.854 \\
\hline $\mathrm{AFP}(\mu \mathrm{g} / \mathrm{L})$ & $10.45 \pm 8.16$ & $11.33 \pm 7.61$ & 0.735 \\
\hline $\mathrm{ALT}(\mathrm{IU} / \mathrm{L})$ & $52.11 \pm 29.96$ & $43.46 \pm 20.40$ & 0.473 \\
\hline $\mathrm{AST}(\mathrm{IU} / \mathrm{L})$ & $64.72 \pm 36.45$ & $53.92 \pm 18.68$ & 0.397 \\
\hline $\mathrm{ALK} \mathrm{P}(\mathrm{IU} / \mathrm{L})$ & $311.44 \pm 215.50$ & $271.5 \pm 138.35$ & 0.831 \\
\hline $\mathrm{INR}$ & $1.34 \pm 0.42$ & $1.29 \pm 0.29$ & 0.656 \\
\hline $\mathrm{TSH}(\mu \mathrm{g} / \mathrm{mL})$ & $4.51 \pm 2.92$ & $4.14 \pm 3.85$ & 0.893 \\
\hline $\mathrm{Bil}($ total $)(\mathrm{mg} / \mathrm{dL})$ & $1.89 \pm 1.33$ & $1.60 \pm 0.83$ & 0.138 \\
\hline $\mathrm{Bil}(\mathrm{direct})$ & $0.59 \pm 0.58$ & $0.61 \pm 0.35$ & 0.326 \\
\hline
\end{tabular}

(BUN: Blood Urea Nitrogen; Cr: Creatinine; Hb: hemoglobin; AFP: alpha-fetoprotein; ALT: Alanine aminotransferase; AST: Aspartate aminotransferase; ALK P: Alkaline Phosphatase; INR: International Normalized Ratio; TSH: Thyroid-Stimulating Hormone; BIL: Bilirubin); Numbers are presented as mean \pm standard deviation.

* Wilcoxon signed-rank test was used to compare the mean before and after intervention

Table (4) : Severity of liver cirrhosis before and after intervention based on MELD scoring system (MELD: model for end-stage liver disease)

\begin{tabular}{|c|c|c|c|}
\hline MELD Score & Before intervention & After intervention & \multirow{2}{*}{$*$ P-value } \\
\hline Average & $11.14 \pm 3.59$ & $12.17 \pm 4.84$ & \multirow{2}{*}{0.552} \\
\hline Median & 11.5 & 11 & \\
\hline Range (Min-Max) & $6-17$ & $6-21$ & \\
\hline
\end{tabular}

Table (5) : Mean score of awareness and quality of life of participants before and after intervention (All of the variables are presented as Mean \pm standard deviation (Min-Max)

\begin{tabular}{|l|c|c|c|c|}
\hline & Before intervention & After intervention & Difference & P-value \\
\hline $\begin{array}{l}\text { Awaren } \\
\text { ess level }\end{array}$ & $4.22 \pm 1.62(0-7)$ & $7.11 \pm 0.83(5-8)$ & $2.88 \pm 0.96(1-5)$ & $0.0001>$ \\
\hline $\begin{array}{l}\text { Quality } \\
\text { of Life }\end{array}$ & $141.89 \pm 20.40(102-170)$ & $182.72 \pm 10.27(167-198)$ & $40.83 \pm 17.73(6-71)$ & $0.0001>$ \\
\hline
\end{tabular}




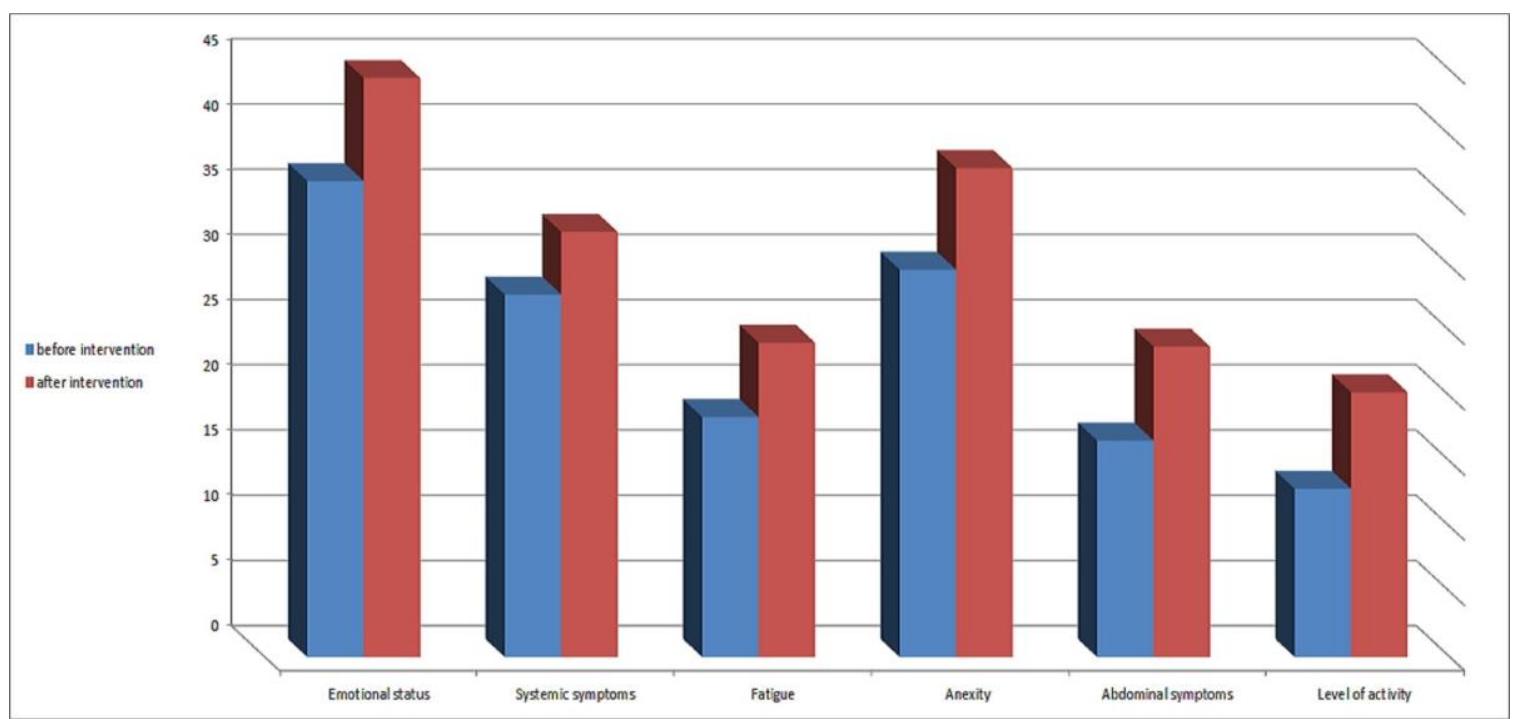

Figure (1) : Comparison of dimensions of quality of life in cirrhotic patients before and after intervention

\section{DISCUSSION}

Patients with chronic liver disease could experience different conditions and need to be aware of disease management course and treatment strategy. Usually in clinical practice, it is not easy for physicians to provide the patients enough information during their routine visiting sessions, and the time allocated to each patient is very limited [17]. The aim of the current study was to investigate the effect of awareness, continuous monitoring and nutritional care on QOL and rate of the liver cirrhosis complications.

The results of this study showed that at the end of the intervention, liver cirrhosis complications, including the rate and severity of edema, the incidence of ascites, bleeding from gastrointestinal varices and hepatic encephalopathy decreased compared to the beginning of the study. Moreover, after the completion of the study, the average time of hospitalization decreased significantly. The increase in the level of patient's awareness consequently resulted in the reduction of complications and duration of hospital stay which could be cost effective.

Volk et al. [18] reported that improving the patient's understanding of the condition could significantly reduce the hospital readmissions among patients with decompensated cirrhosis. In addition, by increasing the level of awareness of patients, they may benefit from better clinical outcomes. For example, Serper et al. [19] found that patients who receive liver transplant had more adherences to treatment and had less need to refer to the hospital and be hospitalized with better knowledge about the treatment regimen.

On the other hand, Wigg et al. [20] investigated the efficacy of a chronic disease management model and demonstrated that the intervention had no significant effect on reducing the number of hospitalization days and the severity of the disease or improving the QOL in patients with chronic liver failure which contradict with the findings of the present study. The reason of this difference could be different study population as well as the type of intervention. In the study by Wigg et al. [20] the intervention was conducted in areas of self-management support, decision support, and clinical information systems; whereas, in the present study, increasing patients' awareness about the disease, nutritional support and continuous monitoring of the patients were conducted for six months.

In the current study, laboratory parameters and severity of liver cirrhosis did not change significantly based on MELD score at the end of intervention though the lack of laboratory improvement should be attributed to the progressive nature of the liver cirrhosis. Moreover, patients' awareness about liver cirrhosis was low at the beginning of the study and after educating and informing, the level of awareness of participants increased significantly (36.11\% rising in awareness). During the study period, patients had been permitted to obtain information from other sources about their condition, which may contribute to increasing the knowledge of

Alavinejad et al., Afro-Egypt J Infect Endem Dis 2019; 9(2): 167-175

https://aeji.journals.ekb.eg/

http://mis.zu.edu.eg/ajied/home.aspx 
patients. Nevertheless, from a practical perspective, this factor has no significant role.

There are limited studies about patients' awareness about liver cirrhosis or the efficacy of a routine educational intervention. In a study by Volk et al. [21], patients' awareness about selfmanagement of liver cirrhosis was reviewed at a U.S.-based health facility. The results indicated a poor awareness level at the beginning of the study and $26 \%$ improvement of knowledge of the patients after a simple nutritional and nonnutrition education intervention for 3 months by using a brief booklet. This increase in awareness about the management of liver cirrhosis is valuable because this intervention was done by using very few resources. However, it was unclear whether this level of awareness would lead to improved outcomes. In another study, Goldsworthy et al. [13] also showed understanding and awareness of patients about liver cirrhosis was poor at the beginning of the study and after using multimedia education and informing patients, the score of patients' awareness questionnaire increased significantly compared to the beginning of the intervention by $41.7 \%$. Therefore, the conducted intervention in this study was an effective way to empower patients with liver cirrhosis. In addition, Kadokawa et al. [17] investigated the effectiveness of conducting chronic liver disease education classes for informing patients with chronic hepatitis and liver cirrhosis. The level of awareness of the participants in the study improved significantly after participating in the classes, moreover, the recovery rate depended on the number of class attendance. These results are consistent with the findings of the present study.

One of the reasons for the poor level of awareness about liver disease in this study, as well as other studies, is difficulty in remembering the information given by the responsible doctors in routine visits; or insufficiency of the provided information. However, these two reasons are not exclusive. Information given in routine clinical visits usually focuses on commonly used treatment regimens as well as alternative therapies that may be implemented in the near future, and less attention is paid to issues such as long-term care and or other details. This is partly due to the limited time assigned for counseling into the routine clinical program. In this situation, patients and their family members may not be aware about the importance of the information presented to them. It should also be noted that it is difficult to measure and correct or change patients' awareness or believes about their disease and treatment due to several interfering factors such as health literacy, beliefs about health, medications and treatment, the relationship between the patient and the physician (such as the quality of education and communication), self-efficacy, and the impact of the other internal and external barriers [22-24]. These barriers may include social or economic factors, physician-related or healthcare-related factors, and patient-related factors, and could potentially affect adherence to treatment [25]. Therefore, further studies are needed to identify the factors affecting patients' awareness and following of the given instructions.

Another finding of this study was improving of the QOL of cirrhotic patients which increased by $11.1 \%$ after intervention. Various studies have shown that protein-energy malnutrition is associated with reduced survival in patients with liver cirrhosis $[\mathbf{7 , 2 6 ]}$. Optimal nutrition status is essential in managing patients with advanced liver disease. Patients with liver cirrhosis are suffering from malnutrition which result in increased morbidity and reduced QOL due to adverse side effects [7]. In a randomized controlled study in India by Maharshi et al. (2016), patients with liver cirrhosis were evaluated in two groups of nutrition support and control for 6 months. The results showed that the QOL associated with health in the intervention group was significantly improved compared to the control group [27]. In another study, Zandi et al. [15] showed that implementing self-care education programs and continuous monitoring of cirrhotic patients for three months would significantly improve the QOL of these patients. These results are consistent with the findings of the present study.

In sum, the results obtained in this study and similar studies show that patients' QOL improves significantly after the intervention by education and informing. Therefore, educational interventions can be used as a useful way to increase the QOL of cirrhotics in clinical setting.

One of the main limitations of this study is the using self-assessment method and no objective measurement to assess the level of awareness. Other limitations of this study include being a single-center study and low number of participants, being a pilot study, lack of long-term follow-up and not considering the control group to compare the effectiveness of intervention results due to 
ethical issues. Among the uncontrollable limits of this study, we can mention the previous knowledge and experience, and the motivation and interest of patients who are effective in implementing the program.

\section{CONCLUSION}

The results of this study showed that patients' awareness about liver cirrhosis could be improved through a simple educational intervention and continuous monitoring for 6 months and would result in fewer side effects, reduced the number of hospitalization days and improved QOL of the patients. By improving the patients' QOL, they would encourage to prevent leaving of treatment. More studies are required to determine the exact impact of more intensive educational interventions on improving results, adhering to the treatment, and lowering medical costs in patients with liver cirrhosis.

\section{Acknowledgement}

This article has been derived from final thesis of Dr Baharak Danyaee for her internal medicine residency course.

\section{Conflict of interest}

The authors declare to have any conflict of interest. While this research involving Human Participants, we did not prescribed them any medicine or supplementary material with potential hazards and all of the participants requested to sign an informed consent before inclusion in study. This study has been done under supervision of Alimentary Tract Research Center without any financial funding and approved by Ahvaz Jundishapur University of Medical Sciences ethical committee (IRAJUMS. REC.1396.811).

\section{REFERENCES}

1. Nusrat S, Khan MS, Fazili J, Madhoun MF. Cirrhosis and its complications: evidence based treatment. World Journal of Gastroenterology: WJG. 2014 May 14;20(18):5442.

2. Williams R, Aspinall R, Bellis M, Camps-Walsh G, Cramp M, Dhawan A et al. Addressing liver disease in the UK: a blueprint for attaining excellence in health care and reducing premature mortality from lifestyle issues of excess consumption of alcohol, obesity, and viral hepatitis. The Lancet. 2014 Nov 29;384(9958):1953-97.
3. Hansen L, Sasaki A, Zucker B. End-stage liver disease: challenges and practice implications. Nursing Clinics. 2010 Sep 1;45(3):411-26.

4. Schuppan D, Afdhal NH. Liver cirrhosis. The Lancet. 2008 Mar 8;371(9615):838-51.

5. Orr JG, Homer T, Ternent L, Newton J, McNeil CJ, Hudson M, Jones DE. Health related quality of life in people with advanced chronic liver disease. Journal of Hepatology. 2014 Nov 1;61(5):115865.

6. Elfaki AM. Aetiology, complications, and preventive measures of liver cirrhosis; Elobeid Hospital; West Sudan. Sudan Journal of Medical Sciences. 2008;3(1):25-8.

7. Trivedi HD, Tapper EB. Interventions to improve physical function and prevent adverse events in cirrhosis. Gastroenterology Report. 2018 Jan 10;6(1):13-20.

8. Huisman EJ, Trip EJ, Siersema PD, van Hoek B, van Erpecum KJ. Protein energy malnutrition predicts complications in liver cirrhosis. European Journal of Gastroenterology \& Hepatology. 2011 Nov 1;23(11):982-9.

9. Barello S, Graffigna G, Vegni E, Bosio AC. The challenges of conceptualizing patient engagement in health care: a lexicographic literature review. Journal of Participatory Medicine. 2014 Jun 11;6(11):259-67.

10. Fagerlin A, Sepucha KR, Couper MP, Levin CA, Singer E, Zikmund-Fisher BJ. Patients' knowledge about 9 common health conditions: the decisions survey. Medical Decision Making. 2010 Sep; 30(5-suppl):35-52.

11. McCartney M. Patient information leaflets:" a stupid system. BMJ. 2013 Mar;347.

12. Valery PC, Powell E, Moses N, Volk ML, McPhail SM, Clark PJ, Martin J. Systematic review: unmet supportive care needs in people diagnosed with chronic liver disease. BMJ Open. 2015 Apr 1; 5(4): e007451.

13. Goldsworthy MA, Fateen W, Thygesen H, Aldersley MA, Rowe IA, Jones RL. Patient understanding of liver cirrhosis and improvement using multimedia education. Frontline Gastroenterology. 2017 Jan 19:flgastro-2016.

14. Younossi ZM, Guyatt G, Kiwi M, Boparai N, King D. Development of a disease specific questionnaire to measure health related quality of life in patients with chronic liver disease. Gut. 1999 Aug 1; 45(2): 295-300.

15. Zandi M, Adib-Hajbagheri M, Memarian R, Nejhad AK, Alavian SM. Effects of a self-care program on quality of life of cirrhotic patients referring to Tehran Hepatitis Center. Health and Quality of Life Outcomes. 2005 Dec;3(1):35. 
16. Chawla KS, Talwalkar JA, Keach JC, Malinchoc $\mathrm{M}$, Lindor KD, Jorgensen R. Reliability and validity of the Chronic Liver Disease Questionnaire (CLDQ) in adults with non-alcoholic steatohepatitis (NASH). BMJ Open Gastroenterology. 2016 Mar 1;3(1): e000069.

17. Kadokawa Y, Katayama K, Takahashi K, Fukushima N, Tanaka S, Taniguchi Y et al. The Effectiveness of a Liver Disease Education Class for Providing Information to Patients and Their Families. Journal of Clinical Medicine Research. 2017 Mar;9(3):207.

18. Volk ML, Tocco RS, Bazick J, Rakoski MO, Lok AS. Hospital readmissions among patients with decompensated cirrhosis. The American Journal of Gastroenterology. 2012 Feb;107(2):247.

19. Serper M, Patzer RE, Reese PP, Przytula K, Koval R, Ladner DP et al. Medication misuse, nonadherence, and clinical outcomes among liver transplant recipients. Liver Transplantation. 2015 Jan 1;21(1):22-8.

20. Wigg AJ, McCormick R, Wundke R, Woodman RJ. Efficacy of a chronic disease management model for patients with chronic liver failure. Clinical Gastroenterology and Hepatology. 2013 Jul 1;11(7):850-8.

21. Volk ML, Fisher N, Fontana RJ. Patient knowledge about disease self-management in cirrhosis. The American Journal of Gastroenterology. 2013 Mar;108(3):302.
22. Horne R, Chapman SC, Parham R, Freemantle N, Forbes A, Cooper V. Understanding patients' adherence related beliefs about medicines prescribed for long-term conditions: a metaanalytic review of the Necessity-Concerns Framework. PloS one. 2013 Dec 2;8(12):e80633.

23. AlHewiti A. Adherence to long-term therapies and beliefs about medications. International Journal of Family Medicine. 2014;2014.

24. Osborn CY, Paasche-Orlow MK, Bailey SC, Wolf MS. The mechanisms linking health literacy to behavior and health status. American Journal of Health Behavior. 2011 Jan 1;35(1):118-28.

25. Hayward KL, Martin JH, Cottrell WN, Karmakar A, Horsfall LU, Patel PJ et al. Patient-oriented education and medication management intervention for people with decompensated cirrhosis: study protocol for a randomized controlled trial. Trials. 2017 Dec;18(1):339.

26. Alberino F, Gatta A, Amodio P, Merkel C, Di Pascoli L, Boffo G, Caregaro L. Nutrition and survival in patients with liver cirrhosis. Nutrition. 2001 Jun 1;17(6):445-50.

27. Maharshi S, Sharma BC, Sachdeva S, Srivastava S, Sharma P. Efficacy of nutritional therapy for patients with cirrhosis and minimal hepatic encephalopathy in a randomized trial. Clinical Gastroenterology and Hepatology. 2016 Mar 1;14(3):454-60. 